\title{
Relación del salto contramovimiento y pruebas de velocidad (10-30 m) y agilidad en jóvenes futbolistas chilenos Relationship of counter-movement jump and speed tests $(10-30 \mathrm{~m})$ and agility in young Chilean soccer players \\ * Joel Barrera, ** LuisValenzuela Contreras, **Álvaro Segueida Lorca, ***Fernando Maureira Cid, **Eduardo Zurita, *Hugo Sarmento \\ *U niversity of Coimbra (Portugal), **U niversidad Católica Silva Henríquez (Chile), ***U niversidad M etropolitana de Ciencias de la Educación (Chile)
}

Resumen. El objetivo de la presente investigación fue determinar la relación que existe entre el sal to contramovimiento y pruebas de velocidad (10-30 m) y agilidad. La muestra estuvo constituida por 169 futbolistas jóvenes entre las categorías sub13 y sub-19, a los cuales se les aplicó la prueba de salto contramovimiento, velocidad (10-30 m) y agilidad zigzag. Los resultados muestran que la altura del salto contramovimiento presenta una alta correlación negativa con la velocidad $10 \mathrm{~m}$ $(r=-.693), 30 \mathrm{~m}(r=-.731)$ y agilidad ( $r=-.490)$. De igual modo, los resultados de la potencia del salto contramovimiento se correlacionan negativamente con la velocidad $10 \mathrm{~m}(\mathrm{r}=-.658), 30 \mathrm{~m}(\mathrm{r}=-.719)$ y agilidad $(r=-.484)$. Los coeficientes de determinación más altos correspondieron a la altura del salto contramovimiento y velocidad $\left(r^{2}=.534\right)$ y la potencia del salto contramovimiento y velocidad $\left(r^{2}=.517\right)$. Los resultados obtenidos en el presente estudio sugieren que el salto contramovimiento tiene un alto grado de influencia en el rendimiento en la velocidad (10-30 m), lo cual, parece no estar tan claro en la variable de agilidad.

Palabras claves: Fútbol, jóvenes, salto contramovimiento, pruebas de campo.

\begin{abstract}
The objective of this research was to deter mine the relationship between countermovement jumping and speed tests $(10-30 \mathrm{~m})$ and agility. The sample consisted of 169 young soccer players between the under- 13 and under- 19 categories, to whom the counter-movement jump test, speed (10-30 m) and zigzag agility were applied. The results show that the height of the countermovement jump shows a high negative correlation with speed $10 \mathrm{~m}(r=-.693), 30 \mathrm{~m}(r=-.731)$ and agility $(r=-.490)$. Similarly, the results of the counter movement jump power arenegatively correlated with speed $10 \mathrm{~m}(r=-.658)$, $30 \mathrm{~m}(r=-.719)$ and agility $(r=-.484)$. The highest coefficients of determination corresponded to the height of the countermovement jump and speed $(r 2=.534)$ and the power of the countermovement jump and speed $(r 2=.517)$. The results obtained in the present study suggest that the countermovement jump has a high degree of influence on speed performance (10-30 m), which seems to be not so clear in the agility variable.
\end{abstract}

Keywords: Football, youth, countermovement jump, field trials.

\section{Introducción}

El fútbol es probablemente el deporte más popular del mundo (Fernández-Rio et al. , 2019) y lo practican hombres y mujeres, niños y adultos con diferentes niveles de experiencia (Stølen et al., 2005). En su natura leza el fútbol, es un deporte de colaboración y oposición, con una participación simultánea y de exigencias atléticas intermitentes (niveles óptimos de fuerza muscular, potencia, velocidad, agilidad y resistencia) que son la base para al canzar un alto desempeño en competición (N egra et al., 2016). En este contexto, la identificación y el desarrollo del talento es un componente

Fecha recepción: 22-10-20. Fecha de aceptación: 15-02-21 oel Barrera

jibarrera@ outlook.es esencial del fútbol profesional moderno (Nevill et al., 2019), actualmente, dicho proceso de identificación y selección de talentos en jóvenes futbolistas masculinos se centra principalmente en la antropometría y el rendimiento físico (Peña-Gonzalez et al., 2018), en este sentido se ha demostrado que los mejores equipos continúan aumentando sus capacidades físicas, mientras que los peores clasificados tienen val ores similares a los reportados hace 30 años (Stølen et al., 2005).

El salto contramovimiento (CMJ), evaluado por medio de una plataformade sal to, esun método confiable y válido para la estimación del poder explosivo de las extremidades inferiores, en el proceso completo de estiramiento acortamiento (Markovic et al., 2004). En la actualidad las habilidades de velocidad y poder se requieren progresivamente en el fútbol contemporáneo para permitir que los jugadores cumplan las demandas de un partido competitivo (Loturco et al., 2018). La 
evidencia sugiere que el aumento de la potencia en lugar de la fuerza puede ser más beneficioso para el mejoramiento en la capacidad de velocidad en la juventud (Hammami et al., 2015).

Por otra parte, se ha demostrado que el porcentaje de tiempo empleado en correr y correr a alta velocidad, en la primera y segunda mitad fue 9.38 y $8.29 \%$ respectivamente (Wehbe et al., 2014). La velocidad máxima es una métrica importante para el proceso de individualización de los futbolistas y generalmente se mide durante una prueba de velocidad, a pesar de que se hayan informado valores más altos en los partidos en comparación con las pruebas de $40 \mathrm{~m}$ en jugadores adultos, pero no en jóvenes futbolistas (Kyprianou et al., 2019). Adicionalmente, Bustos, Acevedo y Rodríguez (2017) encontraron relaciones entre el CMJ y sprint de $20 \mathrm{~m}$ en jóvenes jugadores colombiano ( $r=-.44 ; p$ $<.05)$, mientras que Coelho et al., (2011) también obtuvo correlaciones entre el CMJ la velocidad 10, $20 \mathrm{y}$ $30 \mathrm{~m}$ ( $r=0.293,0.386$ y 0.441 respectivamente) pero con jugadores sub20 y profesionales.

En tanto, la agilidad reactiva y la velocidad de cambio de dirección son determinantesimportantes del éxito en el fútbol juvenil (Fiorilli et al., 2017; Krolo et al., 2020; Pojskic et al., 2018). La agilidad es una acción de al ta velocidad que implica un cambio rápido de dirección en respuesta a un estímulo (Sheppard \& Young, 2006). Durante el juego, los atletas realizan sprints repetidosqueinvolucran movimientos multidireccionales, en tanto, la mayoría de los giros y vueltas no se planifican previamente, los cuales son de carácter espontaneo y en respuesta a los estímulos externos, como la trayectoria del balón, oponentes y situaciones dinámicas del juego (Born et al., 2016).

Con base en lo anterior y producto de la importancia del desarrollo de las capacidades físicas en el rendimiento deportivo, el propósito de este estudio fue exa minar y determinar las correlaciones entre el CMJ y la velocidad (10 - $30 \mathrm{~m}$ ) y agilidad. Esto es importante ya que (I) permitirá determinar si existe relación entre el CMJ y el rendimiento de las pruebas de velocidad, (II) los resultados suponen una herramienta útil para la utilización de entrenadores que se desenvuelven en el proceso formativo y (III) la producción científica con rela ción a los jóvenes futbolistas en Chile.

\section{Metodología}

\section{Muestra}

Lamuestra estuvo constituida por jóvenes futbolistas chilenos de las categorías Sub-13, Sub-14, Sub-15, Sub16, Sub-17 y Sub-19 de dos equipos profesionales de primera división $\mathrm{N}=169$; edad $=15.55 \pm 2.06$ años; masa corporal $=63.18 \pm 9.87 \mathrm{~kg}$; altura $=170.98 \pm 8.40$ $\mathrm{cm}$. Como criterio de exclusión se consideró ser miembros activos del torneo nacional. Por ser atletas menores de edad se debió aplicar un consentimiento informado para cada atleta el cual debía ser firmado por su representante legal. Los procedimientos experimenta les de este estudio estaban adscritos con la Declaración de Helsinki.

\section{Instrumentos}

Para las pruebas de velocidad (10-30 m) y agilidad, se utilizaron fotocélulas (Smartspeed Pro, Additional Gate, fusion sport ${ }^{\circledR}$ ) y para la prueba de CM J, se utilizó una plataforma de salto de la misma marca. Por último, para la medición de la altura y el peso se utilizó un estadiómetro fijado a la pared $\left(360^{\circ} \mathrm{W}\right.$ ireless Seca 284 dp, Alemania) y para la obtención del peso total se utilizará una balanza digital balanza (Avanutri modelo BL MB con una división de $50 \mathrm{gr}$ ).

\section{Procedimiento}

La colecta de datos se llevó a cabo durante la temporada 2018-2019. Los futbolistas, previo a cada prueba física debían realizar un calentamiento general $10 \mathrm{mi}$ nutos y uno específico el cual se ajustaba a la capacidad a ser evaluada. En las diferentes evaluaciones, los atletas realizaron tres intentos de los cuales se consideró el mejor de ellos.

Para el salto vertical contramovimiento (CMJ) el atleta se ubicó sobre la plataforma en posición bípeda y los pies separados a la anchura de los hombros, a la señal el deportistadebió realizar unaflexión de rodillasaproximada de $90^{\circ}$ y de manera inmediata un sal to máximo en la vertical que se encuentra, durante la etapa de vuelo el deportista no debió flexionar las caderas o las rodillas, ya que para que el salto sea válido se deben mantener extendidas durante el vuelo y el operador lo comprobó visualmente (Gugu-Gramnatopol et al., 2017). El salto se debe ejecutar con las manos en la cintura y no podrá retirarlas de esa posición durante la ejecución del procedimiento, con la finalidad de obtener un resultado que refleje solo la fuerza de los miembros inferiores sin el aporte que pueda generar el impulso de los brazos (M arkovic et al., 2004). Cada atleta tendrá la posibilidad de realizar tres sal tos con un período de recuperación de al menos 30 segundos entre cada uno de ellos, con el objeto de no mermar el rendimien- 
to entre cada uno de los saltos, considerando que la vía en anaeróbica láctica puede sostener actividades de máximo esfuerzo entre 6 y 10 segundos (Chicharro \& Mulas, 1996) y, donde cadasalto puede durar aproximadamente un segundo. Los datos utilizados de esta eva luación fueron la altura y la potencia mediante la ecua ción propuesta por Sayers et al. (1999):

Potencia $(W)=51.9 \times C M J$ altura $(\mathrm{cm})+48.9 \times$ masa corporal (kg) - 2007

Para la evaluación de la velocidad (segundos) se utilizó la prueba de 10 y 30 m en línea recta, para esto fueron necesario tres pares de fotocélulas, al inició de la prueba, a los $10 \mathrm{~m}$ y a los $30 \mathrm{~m}$, la distancia más larga del recorrido, parámetros que ya han sido utilizados en evaluaciones anteriores en futbolistas (Little $\&$ W illiams, 2005; M alý et al., 2015). Los jugadores comenzaron la prueba en posición bípeda a una distancia de dos metros antes de la primera fotocélula. Para este procedimiento los jugadores deberán realizar tres sprint, considerando una recuperación pasiva de tres minutos entre cada uno de ellos (Köklü et al., 2015). Cada procedimiento será estimado para ambas pruebas $10 \mathrm{~m}$ y $30 \mathrm{~m}$ respectivamente con la finalidad de optimizar los tiempos de ejecución, de las cuales se considerará el mejor tiempo de ejecución registrado por cada atleta.

La prueba de agilidad en Zig-Zag, propuesto por Little y W illiams (2005) y utilizado por Kunrath et al. (2017) el cual consiste en realizar un recorrido de cua tro secciones de cinco metros con un ángulo de $100^{\circ}$ y un recorrido total de $20 \mathrm{~m}$. Está prueba se recomienda, debido a que utiliza constantes aceleraciones, frenos y cambios de dirección tal como ocurre en la práctica deportiva. Los atletas debieron estar en posición bípeda un metro antes de la posición del inicio de la prueba donde se instaló el primer par de fotocélulas y el segundo al final del recorrido, luego se dio una señal sonora para que los jugadores comenzarán su recorrido, el cual podían realizar en tres oportunidades con una recuperación pasiva de tres minutos entre cada prueba.

\section{Análisis estadísticos}

Para el análisis de datos se utilizó el programa esta dístico IBM SPSS 25.0. Los primeros indicadores descriptivos que se extrajeron de los datos fueron las medias y desviaciones estándar. La normalidad de la distribución de datos se verificó mediante la prueba de Kolmogorov-Smirnov (M aureira, 2017). Para verificar la existencia de una correlación bivariada entre el CMJ y la velocidad (10-30 m) y agilidad, se utilizará el esta dístico de Pearson, para lo cual se adaptaron los siguien- tes criterios ya sean positivos o negativos para interpretar la magnitud de la correlación ( $r$ ) entre las medidas de prueba: <0.1 trivial, 0.1-0.3 pequeño, 0.3-0.5 moderado, 0.5-0.7 grande, 0.7-0.9 muy grande y 0.91.0, casi perfecto (Bonilla et al., 2020). Finalmente, se realizó una regresión lineal simple para determinar el coeficiente de determinación $\left(r^{2}\right)$ entre el CMJ y la velocidad (10-30 m) y agilidad (Little \& W illiams, 2005; Maly et al., 2014).

\section{Resultados}

Los resultados de las medias y desviaciones estándar de las variables de edad, masa corporal, altura, CMJ (altura y potencia), velocidad (10 - $30 \mathrm{~m}$ ) y agilidad (zig-zag) se muestran en la tabla 1. Por otra parte, en la tabla 2, se presentan los resultados de las correlaciones bivariadas de Pearson entre el CMJ (altura) y las pruebas ya señaladas de los atletas por categorías. En la tabla 3, se muestran la relación entre el CMJ (potencia) y las mismas variables.

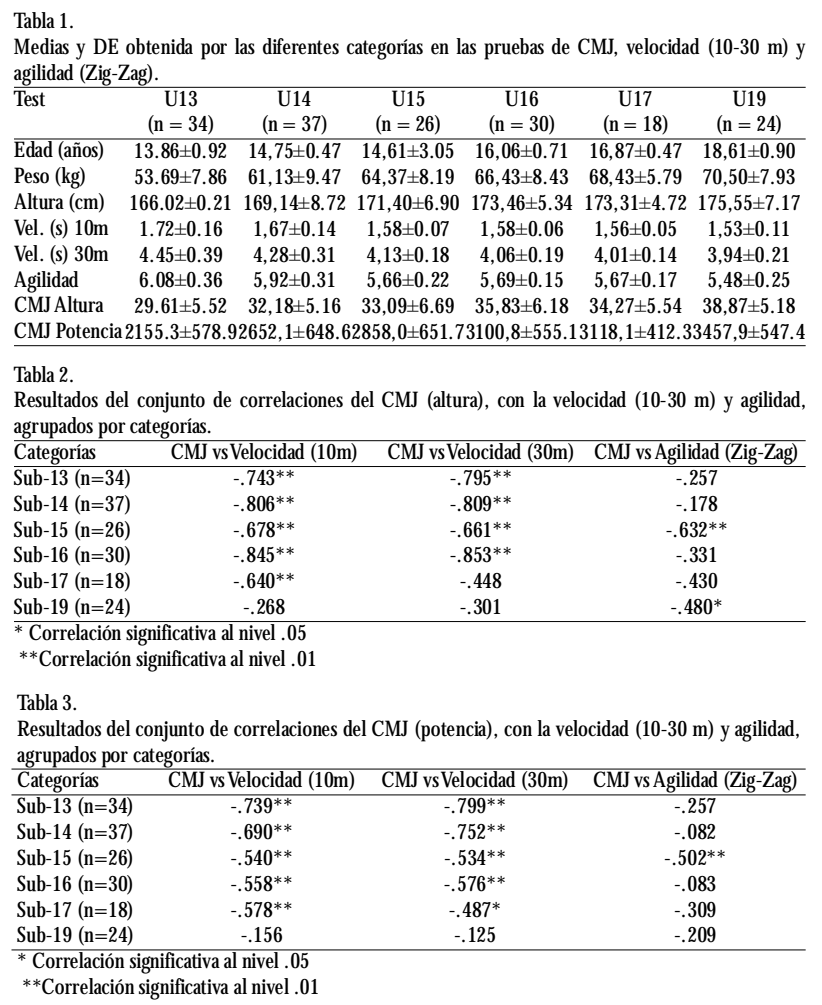

Al considerar el total de la muestra, se puede observar diferentes tipos niveles de correlaciones negativas entre el CMJ (altura) y las pruebas de velocidad $10 \mathrm{~m}$ (grande), $30 \mathrm{~m}$ (muy grande) y agilidad (moderada) (tabla 4), en un nivel de significancia de $(P<.01)$. De igual modo, los resultados de correlación entre el CMJ (potencia) y las pruebas ya señaladas, arrojo resultados similares en cada una de las variables, los cuales se re- 
flejan en la tabla 5. Todos estos datos al canzaron un nivel de significancia de $(P<.01)$.

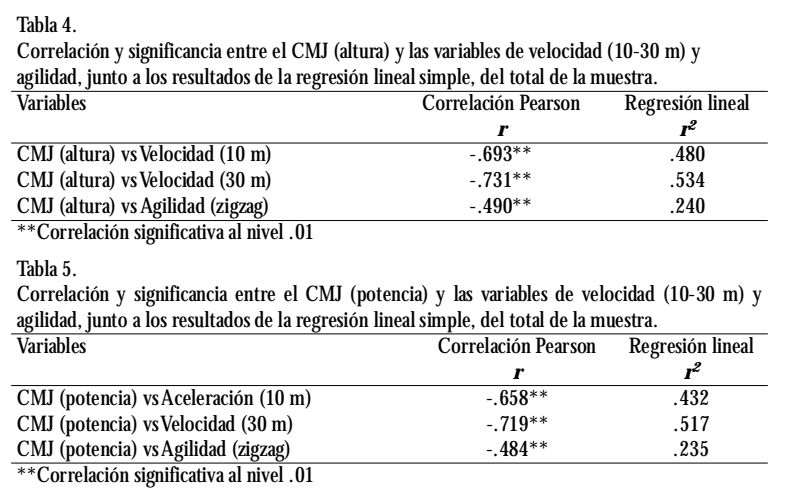

Por otra parte, el coeficiente de determinación de la regresión lineal simple del CMJ (altura) con las va riables de velocidad $10 \mathrm{~m}\left(\mathrm{r}^{2}=.480\right), 30 \mathrm{~m}\left(\mathrm{r}^{2}=.534\right) \mathrm{y}$ agilidad $\left(r^{2}=240\right)$ y del CMJ (potencia) con las mismas variables entregan algunos valores cercanos al $50 \%$ $\left(r^{2}=.432 ; r^{2}=.517 ; r^{2}=.235\right.$, respectivamente).

No se encontraron correlaciones significativas del CMJ (altura) con la velocidad $10 \mathrm{~m}$ en la categoría Sub19, con la prueba de velocidad $30 \mathrm{~m}$ en las categorías Sub-17 y Sub-19, y con la prueba de agilidad en las categorías Sub-13, Sub-14, Sub-16 y Sub-17. Por otra parte, no se mostraron correlaciones significativas del CMJ (potencia) con las pruebas de velocidad (10 - $30 \mathrm{~m}$ ) y agilidad en la categoría Sub-19 y con la prueba de agilidad en las categorías Sub-13, Sub-14, Sub-16 y Sub-17.

\section{Discusión}

El objetivo del presente estudio fue determinar la relación del CMJ (altura y potencia) con las pruebas de velocidad (10-30 m) y agilidad (zigzag). El hallazgo principal es que se encontraron relaciones significativas entre los indicadores de rendimiento futbolístico considerados en este estudio, al igual que Köklü et al., (2015), lo cual, tal como sugieren estos autores la velocidad (10-30 m), la agilidad y la capacidad de salto vertical, comparten determinantes fisiológicos (tipo de fibra muscular y sistema energético ATP-PC) y biomecánicos comunes (Köklü et al., 2015, Little \&W illiams, 2005). Sin embargo, otros autores como Harris et al. (2008) y Little yW illiams (2005) indicaron que, en adultos, tra bajos en distancias cortas de $10 \mathrm{~m}$ y largas de $30 \mathrm{~m}$ se requieren capacidades neuromusculares y biomecánicas separadas y específicas para cada una de ellas. Lo señala do anteriormente, a pesar de ser en atletas adultos, pa rece tener más relación con los resultados presentados en este estudio, ya que, a pesar de tener en ambos indicadores correlaciones significativas, los resultados en la regresión lineal fueron más altos en velocidad 30 m, lo cual sugiere que tanto las cualidades biomecánicas y neuromusculares son diferentes en estas pruebas, a pesar de compartir determinantes fisiológicos.

Al considerar el total de la muestra, los resultados del estudio arrojaron una correlación negativa grande, muy grande y moderada entre el CMJ (altura y potencia) con la velocidad (10-30 m) y agilidad respectiva mente, con un nivel de significancia de $(p<0.001)$. Sin embargo, al fragmentar el total de la muestra en las diferentes categorías, los resultaos demostraron ser menos concluyentes. Adicionalmente, los coeficientes de determinación muestran que las pruebas con los índices de correlación más significativos, CMJ (altura y potencia) y velocidad, comparten una predicción del $53.4 \%$ y $51.7 \%$ respectivamente, tal como se refleja en las tablas 4 y 5 , lo cual sugiere que a medida que aumenta la distancia de sprint la relación entre las va riables se vuelve más fuerte, es decir, si bien la correlación es significativa en los 10 m (grande), esta aumenta en los 30 m (muy grande), tal como ocurrió con la resultados de Köklü et al., (2015) y Coelho et al., (2011). Esto podría explicarse, ya que ambos estudios fueron realizados jóvenes futbolistas de élite, lo cual, a su vez, permite inferir una similitud entre distintas poblaciones del mismo rango etario, tal como aconteció en la comparación de jugadores colombianos con publicacionesinternacionales (Rojaset al. , 2020). En tanto,W isløff et al., (2004) en jugadores profesionales de élite (Trondheim, Noruega), en $10 \mathrm{~m}$ presento peores rendimientos que los jóvenes futbolistas chilenos (1.83 s), sin embargo, en los $30 \mathrm{~m}$, al canzaron un mejor registro (4.0 s), tales diferencias se pueden explicar al ser juga dores de diferentes niveles etarios y de competición (H augen, Tonnessen \& Seiler, 2012), que por ende tiene un desarrollo físico superior, necesarios para competir en una liga profesional.

En relación a la existencia de correlación entre la fuerza muscular de las extremidades inferiores, por medio del salto horizontal y la velocidad, Almeida, Marques de M elo, y Souza (2016) obtuvieron datos de clasificación débil negativa con jugadores de fútbol Sub13, los cuales difieren con los obtenidos en el presente estudio. lo que se puede deber a la diferencia de la prueba de salto y velocidad utilizados. En este mismo sentido algunos autores intentaron determinar la contribución de la fuerza explosiva vertical con el rendimiento de sprint en niños y establecieron una correlación moderada entre brazo-CMJ y el sprint de $30 \mathrm{~m}(r=.40)$, sin 
embargo, la correlación entre brazo-CMJ y los 20m fue grande ( $r=.52)$ (Hammami et al., 2015). Los resultados de estas correlaciones fueron más bajos que los obtenidos en velocidad ( 10 - $30 \mathrm{~m}$ ) en la categoría Sub-13 que es el rango etario más próximo a los del estudio en cuestión, estos resultados pueden ser objeto de la diferencia de edad, así como la práctica deportiva constantes de los sujetos de este estudio, o por la existencia del efecto de edad relativo existente en los jóvenes jugadoreschilenos (Barrera, Valenzuela, M aureiray Sarmento, 2020), que a su vez está asociado a una ventaja en el rendimiento físico debido al mes de nacimiento y al estado de maduración biológica (Peña-González et al., 2018), lo que refleja la selección sistemática y retención de jugadores avanzados en estado de madurez, y la exclusión de jugadores con madurez tardía comenzando alrededor de los 12-13 años de edad (Malina, Figueiredo \& Coelho-e-Silva, 2017) lo que podría explicar la diferencia en el rendimiento de jugadores del mismo rango etario.

Por otra parte, un estudio realizado con futbolistas Sub-21 de élite, obtuvo peores valores a los de este estudio en las variables de velocidad $10 \mathrm{~m}(1.77 \pm 0.10)$ y $30 \mathrm{~m}$ (4.23 \pm 0.19$)$, mientras que en la prueba de CMJ (altura) los resultados fueron mayores (Coratella, Beato, $\&$ Schena, 2018), pero losautores no correlacionaron estos rendimientos para saber si el comportamiento era similar a los de este artículo. Los descubrimientos de este estudio se correlacionan significativamente entre estas variables, es decir, una mayor altura en la prueba de CMJ, implica un menor tiempo en cada una de las pruebas de velocidad, lo que se traduce a sujetos más rápidos. Es necesario aclarar, que la relación que se pretende explicar en el presente estudio en relación al CMJ, está afín con las pruebas de velocidad $(10-30 \mathrm{~m})$ y agilidad, lo cual no explica el rendimiento técnico (juego aéreo) que pueda o no tener un jugador durante el desarrollo del juego alcanzado más o menos altura en la prueba de salto, ya que, requiere de otros elementos como la fuerza, la agilidad y la rapidez de ejecución, las cuales facilitan este proceso de elevación (GuguGramnatopol et al., 2017). Por lo cual, las pruebas de sal tos son un indicador cuantitativo de rendimiento físico de los jóvenes futbolistas y su abordaje debe ser específico en esta materia.

En tanto, los niveles de fuerza pico relativos al CMJ se asociaron significativamente con la cinética de sprint (potencia y fuerza horizontal) y tiempos de sprint de 5$20 \mathrm{~m}(r=-.589$ a $r=-.630)$ y la altura del salto y el tiempo concéntrico en el CMJ se correlacionaron significativamente con el tiempo de sprint a 20m ( $r=-$ $.550 \mathrm{y} r=-.546$ ) respectivamente (M orris et al., 2020). Estas relaciones son próximas a las obtenidas en este estudio al compararla con la velocidad (10-30 m) a pesar de ser atletas adultos, estos sugiere que los resulta dos obtenidos pueden deberse a la cantidad de horas destinadas a mejorar la condición física de los jugadores jóvenes en el proceso de formación (Metaxas et al., 2019).

Por otra parte, se ratifica la existencia de relación entre la fuerza máxima y la velocidad y el rendimiento en salto, en jugadoresprofesionales (W isløffet al., 2004). Pero estos autores no obtuvieron el coeficiente de determinación, lo cual podría haber resultado interesante para ver la incidencia de la fuerza máxima en las variables analizadas, ya que, tal como demostró Little y W illiams (2005) al evaluar a 106 jugadores de fútbol profesionales que fueron evaluados para velocidad 10 $\mathrm{m}$, sprint de $20 \mathrm{~m}$ de vuelo (velocidad máxima) y rendimiento de agilidad, existen correlaciones significativamente entre las tres pruebas $(p<.0005)$, sin embargo, los coeficientes de determinación $\left(r^{2}\right)$ fueron solo del 39, 12 y $21 \%$ para la aceleración y la velocidad máxima, la aceleración y la agilidad, y la velocidad y agilidad máximas, respectivamente. Lo cual deja un margen entre las relaciones que puede ser explicado por otras variables como edad, nivel de maduración, años de entrenamiento entre otras.

Final mente hay que destacar que la literatura propone que los métodos de entrenamiento enfocados en la pliometría (vertical u horizontal) puede producir mejoras en el salto vertical, agilidad y velocidad (M anouras et al. , 2016), así como losestudiosenfocados en la potencia máxima relativa (M orris et al., 2020) y a los entrenamientos de fuerza explosiva (Hammami et al. , 2015). Sin embargo es importante señalar que debe existir un foco específico en mejorar la potencia pico relativa y en entornos de tiempo limitado para los deportes colectivos (M orris et al., 2020). Además, se propone considerar individualmente cada una de estas ca pacidades al momento de diseñar planes de entrenamientos para los jóvenes futbolistas, tal como sugieren (Cabell et al., 2019; Coratella et al., 2018; Little \& W illiams, 2005), dado que, a pesar de las correlaciones significativas, los coeficientes de determinación no pueden explicar todo el fenómeno al igual que en este estudio, por lo cual, se sugiere adicional mente a los progra mas de entrenamiento enfocados en la pliometría, programas que se enfoquen en la especificidad del deporte con trabajos que involucren saltos, aceleraciones, fre- 
nos, cambios de dirección y sprint, todas acciones frecuentes en el fútbol moderno y de gran importancia en el rendimiento.

\section{Conclusiones}

EI CMJ es una herramienta valida y muy utilizada para determinar el rendimiento físico en el fútbol y su uso es altamente documentado. Los hallazgos del presente estudio indicaron (I) correlación negativa significativa obtenidas entre las variables analizadas en este estudio sugieren que el CMJ (altura y potencia) tiene un grado de influencia en las variables de velocidad (10 - $30 \mathrm{~m}$ ), es decir, a mayor altura o potencia alcanzada los sujetos de este estudio son más rápidos, ya que, precisan de menos tiempo para realizar los recorridos de las diferentes pruebas; (II) a pesar de las correlaciones existentes entre el CMJ (altura y potencia) y agilidad, se sugiere prudencia para utilizar el CMJ como un elemento que explica el rendimiento en la agilidad, ya que la regresión fue la más baja de este análisis; (III) en virtud de los hallazgos de este estudio que proporcionan evidencia específica para los entrenadores, se sugiere que los programas de entrenamientos que diseñen los especialistasincorporen lapliometría como un el emento preponderante paralamejorade estas variables, así como trabajos de fuerza, pero adicional mente crear estructuras que respondan a la especificidad deportiva incorporando ejercicios que reúnan acciones de salto, aceleraciones, frenos, cambios de dirección y sprint; (IV) finalmente este artículo, pretende aportar al desarrollo y crecimiento de esta disciplina deportiva, con métodos de evaluación y análisis poco utilizado por los departamentos físicos de los clubes formadores en Chile.

\section{Limitaciones}

Las limitaciones en este estudio están enfocadas principalmente a la muestra, la cual corresponde solo a jóvenes futbolistas y puede no ser referencia para otros deportes, así como para categorías adultas. Por lo cual, se propone diseñar estudios que incorporen jugadores profesionales, para determinar si estos resultados se pueden utilizar en jugadores adultos.

\section{Referencias}

Almeida,T., MarquesdeM elo, L., \& Souza, A. (2016). Strength of thecorrelation of lower limbsand speed on the field of football playersunder 13 in the city of Barretos-SP. Revista Brasilera D e Futsal E Futebol, 8(30), 269-274.
Barrera, J., Val enzuela, L., M aureira, F., \& Sarmento, H. (2021). Análisisdeloscomponentesfísicosy antropométricosdejóvenesfutbolistas chilenos desdelacategoríaSub-13 a Sub-19. Retos: nuevastendenciasen educación física, deportey recreación, (39), 108.

Bonilla, A.A.V.,Timon, R., Camacho-Cardenosa, A., CamachoCardenosa, M., Guerrero, S. \& O Icina, G. (2020). Fatigue increases in resting muscle oxygen consumption after a women's soccer match. International Journal of SportsM edicine. https:/ / doi:10.1055/ a 1177-0849

Born, D.-P. , Zinner, C., Dueking, P., \& Sperlich, B. (2016). Multidirectional sprint training improveschange-of-direction speed and reactive agil ity in young highly trained soccer players. Journal of SportsScienceand M edicine, 15(2), 314-319.

Bustos-Viviescas, B. J., Acevedo-M indiola, A. A ., \& RodríguezAcuña, L. E. (2017). Relación entreel salto vertical yel rendimiento delavelocidad enjóvenesfutbolistas. E-motion: Revista deEducación, Motricidad elnvestigación, 9(201), 13-24.

Cabell, L., Zahalka, F., M aly,T., \& Mala, L. (2019). Correlation between sprint tests and agility 505 test and ktest in elite young soccer players. Medicineand Sciencein Sportsand Exercise, 51(6), 201-201. https:// doi:10.1249/ 01. mss 0000561107.31414 .10

Chicharro, J. L., \& Mulas, A. L. (1996). Fundamentosdefisiología del ejercicio. Edicionespedagógicas.

Coelho, D. B., M artins Coelho, L. G., Braga, M. L., Paolucci, A., TorresCabido, C. E., FerreiraJ unior, J. B. , .. \& Garcia, E. S. (2011). Correlation between performance of soccer players in the 30-meter sprint test and in the vertical jump test. M otriz-revista de educacao fisica, 17(1), 63-70.

Cordella, G., Beto, M ., \& Schena, F. (2018). Correlation between quadricepsand hamstringsinter-limbstrength asymmetry with change of direction and sprint in $\mathrm{U} 21$ elite soccer players. Human M ovement Science, 59, 81-87. https:/ / doi.org/ 10.1016/ j.humov.2018.03.016

Fernández-Rio, J., Santos, L., Fernandez-Garcia, B., Robles, R., Casquero, I., \& Paredes, R. (2019). Effectsof slacklinetraining on acceleration, agility, jump performanceand postural control in youth soccer players. Journal of H uman Kinetics, 67 (1), 235-245. https: / / doi. org/ 10.2478/ hukin-2018-0078

Fiorilli, G., Iuliano, E., Mitrotasios, M., Pistone, E. M. , Aquino, G., Calcagno, G, \& di Cagno, A. (2017). A rechange of direction speed and reactive agil lity useful for determining theoptimal field position for young soccer players? J ournal of SportsScience and M edicine, 16(2), 247-253.

Gugu-Gramnatopol, C., O chiotan, S., Ionescu, A. \& Dancescu, D. (2017). Correlation between speed and height jump in junior football players. In E. Soare \& C. Langa(Eds.), EduWorld 7th International Conference(pp. 883-889).

Hammami, R., Makhlouf, I., Chtara, M., Padulo, J., \& Chaouachi, A. (2015). The contribution of vertical explosivestrength to sprintperformancein children. Sport Sciencesfor Health, 11(1), 37-42.

Harris, N. K., Cronin, J. B., Hopkins,W. G., \& Hansen, K.T. (2008). Relationship between sprint timesand the strength/ power outputs of a machine squat jump. The Journal of Strength \& 
Conditioning Research, 22(3), 691-698.

Haugen, T. A., Tønnessen, E., \& Seiler, S. (2012). Speed and countermovement-jump characteristics of elitefemalesoccer players, 1995-2010. International journal of sports physiology and performance, 7(4), 340-349.

Köklü,Y,,Alemdarođlu, U.,ÖZzan,A., Koz, M., \& Ersöz, G. (2015). The relationship between sprint ability, agility and vertical jump performance in young soccer players. Science\& Sports, 30(1), el-e5.

Krolo,A., Gilic, B., Foretic, N., Pojskic, H., Hammami, R., Spasic, M., Uljevik, O.,Versic, S. \& Sekulic, D. (2020). Agility testing in youth football (soccer) players; evaluating reliability, validity, and correlates of newly developed testing protocols. International Journal of Environmental Research and PublicH ealth, 17(1). https: / / doi.org/ 10.3390/ ijerph17010294

Kunrath, C., Gonçalves, E., Teoldo, I. , \& Barbosa, M. (2017). M a duración somáticay aptitud físicaen jóvenesjugadoresdefútbol. RevistaAndaluza deM edicina del Deporte, 10 (4), 187-191.

Kyprianou, E., Di Salvo,V., Lolli, L.,Al Haddad, H.,Villanueva, A . M., Gregson, W., \& Weston, M. (2019). To measure peak velocity in soccer, let the players sprint. Journal of Strength and Conditioning Research. https:/ / doi.org/ 10.1519/ jsc.0000000000003406

Little, T., \&W illiams, A. G. (2005). Specificity of acceleration, maximum speed, and agility in professional soccer players. Journal of Strength and Conditioning Research, 19(1), 76-78. https:/ / doi.org/ 10.1519/ 14253.1

Loturco, I., Pereira, L. A., Kobal, R., \& Nakamura, F.Y. (2018). Using loaded and unloaded jumpsto increasespeed and power performancein eliteyoung and senior soccer players. Strength and Conditioning Journal, 40 (3), 95-103. https:/ / doi. org/ $10.1519 /$ ssc. 0000000000000249

Malina, R. M., Figueiredo, A. J., \& Coelho-e-Silva, M. J. (2017). Body size of mal eyouth soccer players: 1978-2015. Sports Mediane, 47(10), 1983-1992.

Malý, T., Zahálka, F., Hráský, P., Mala, L., Izovská, J., Bujnovský, D., Dragijsky, M ., \& Mihal, J. (2015). Age-related differences in linear sprint and power characteristicsin youth elite soccer players. Journal of Physical Education and Sport, 15(4), 857.

Maly,T.,Zahálka, F., Malá, L., \&Teplan,J. (2014). Profile, correlation and structure of speed in youth elite soccer players. Journal of Human Kinetics, 40(1), 149-159.

Manouras, N. , Papanikolaou, Z., Karatrantou, K., Kouvarakis, P., $\&$ Gerodimos,V. (2016).The efficacy of vertical vs, horizontal plyometric training on speed, jumping performanceand agility in soccer players. International Journal of Sports Science \& Coaching, 11(5), 702-709. https:// doi.org/ 10.1177/ 1747954116667108

Markovic, G., Dizdar, D., Jukic, I., \& Cardinale, M. (2004). Reliability and factorial validity of squatand counter movement jump tests. Journal of Strength and Conditioning Research, 18(3), 551-555. https: / / doi.org/ 10.1519/ 00124278-20040800000028

Maureira, F. (2017). Estadística para educación física. Bubok Publishing.
M etaxas, T., M androukas, A., Michailidis, Y., Koutlianos, N., Christoulas, K., \& Ekblom, B. (2019). Correlation of FiberTypeComposition and Sprint Performance inYouth Soccer Players. Journal of Strength and Conditioning Research, 33 (10), 2629-2634. https/ / doi.org/ 10.1519/ jsc.0000000000003320

M orris, C. G.,Weber, J.A., \& N etto, K. J. (2020). Relationship Between Mechanical Effectivenessin Sprint Running and Force-velocity characteristics of a countermovement jump in australian rules football athletes. Journal of Strength and Conditioning Research. https:/ / doi.org/ 10.1519/ jsc.0000000000003583

Negra,Y., Chaabene, H., Hammami, M., Hachana,Y., \& Granacher, U. (2016). Effects of high-velocity resistancetrainingon athletic performance in prepuberal male soccer athletes. Journal of Strength and Conditioning Research, 30 (12), 3290-3297. https:/ / doi.org/ 10.1519/ jsc.0000000000001433

N evill,A. M., O kojie, D. I., Smith, J., O 'D onoghue, P. G., \&Webb, T. (2019). Are professional footballersbecoming lighter and moreectomorphic? Implicationsfor talent identification and development. International Journal of SportsScience\& Coaching, 14(3), 329-335.

Peña_Gonzalez, I., Fernandez-Fernandez, J., M oyazRamon, M. \& \& Cervello, E. (2018). Relative age effect, biological maturation, and coaches' efficacy expectationsin young malesoccer players. Research Quarterly for Exerciseand Sport, 89 (3), 373-379. https:/ / doi.org/ 10.1080/ 02701367.2018.1486003

Pojskic, H.,Aslin, E., Krolo, A. , J ukic, I., Uljevic, O., Spasic, M ., \& Sekulic, D. (2018). Importance of reactive agil ity and change of direction speed in differentiating performance levels in junior soccer players: reliability and validity of newly developed soccer-specific tests. Frontiersin Physiology, 9. https: / / doi.org/ 10.3389/ fphys. 2018.00506

Rojas, I. A. S. , Jiménez, L. E. C., Gutiérrez,Y. P.A., Gálvez,A.J., \& Buitrago, P.J. M. Relación entremarcadoresdermatoglíficos y el perfil morfofuncional en futbolistas profesionales de Bogotá, Colombia (Relationship between dermatoglyphic markersand morphofunctional profilein professional soccer playersfrom Bogotá, Colombia). Retos, (41), 182-190.

Sayers, S. P., Harackiewicz, D.V., Harman, E. A., Frykman, P. N. , \& Rosenstein, M.T. (1999). Cross-validation of threejump power equations. Medicine\& Sciencein Sports\& Exercise, 31 (4), 572-577. Sheppard, J. M ., \&Young, W. B. (2006). Agil lity literature review: Classifications, training and testing. Journal of Sports Sciences, 24(9), 919-932.

Stølen, T., Chamari, K., Castagna, C., \& W isløff, U. (2005). Physiology of soccer. SportsM edicine, 35(6), 501-536.

Wehbe, G. M., H artwig, T. B. \& D uncan, C. S. (2014). M ovement analysis of Australian national league soccer playersusing global positioning system technology. The Journal of Strength \& Conditioning Research, 28(3), 834-842. https:/ / doi.org/ 10.1519/ JSC.0b013e3182a35dd1

W isløff, U., Castagna, C., H elgerud, J., Jones, R., \& H off,J. (2004). Strong correlation of maximal squat strength with sprint performance and vertical jump height in elitesoccer players. British Journal of SportsM edicine, 38 (3), 285-288. https: / / doi.org/ 10.1136/ bjsm.2002.002071 